Article

\title{
Determination of 2-Thioxo-3-pyrrolidinecarbaldehyde in Salted Radish Root (Takuan-zuke) by High- Performance Liquid Chromatography with Fluorescence Detection after Pre-Column Derivatization Using 4-( $N, N-$ dimethylaminosulfonyl)-7-hydrazino- 2,1,3-benzoxadiazole
}

\author{
Taito Kobayashi ${ }^{1}{ }^{(D)}$, Kei Kumakura ${ }^{1}$, Wataru Kobayashi ${ }^{1}$, Asaka Takahashi ${ }^{2}$ \\ and Hiroki Matsuoka ${ }^{1, *}$ \\ 1 Department of Health and Nutrition, Takasaki University of Health and Welfare, 37-1 Nakaorui-machi, \\ Takasaki-shi, Gunma 370-0033, Japan; 1610501@takasaki-u.ac.jp (T.K.); kumakura@takasaki-u.ac.jp (K.K.); \\ 1710302@takasaki-u.ac.jp (W.K.) \\ 2 Higashinihon College of Nutrition and Pharmaceutical, 1098-1 Koyahara-machi, Maebashi-shi, \\ Gunma 379-2184, Japan; asaka@ns.yamasaki.ac.jp \\ * Correspondence: matsuoka@takasaki-u.ac.jp; Tel.: +81-027-352-1290
}

Received: 25 October 2017; Accepted: 24 November 2017; Published: 28 November 2017

\begin{abstract}
TPC) is an important intermediate in the yellowing of Japanese pickles "takuan-zuke". TPC has been reported to have antibacterial activity against bacteria causing food poisoning and microorganisms associated with the development of caries, as well as various physiological functions such as antimutagenicity. However, since TPC has high reactivity, robust quantitative analysis is difficult with the extraction method or pre-column derivatization method using 2,4-dinitrophenylhydrazine. In this study, a high-performance liquid chromatography (HPLC) method involving labeling with 4-(N,N-dimethylaminosulfonyl)-7hydrazino-2,1,3-benzoxadiazole (DBD-H) was developed for the determination of the level of TPC in takuan-zuke. DBD-TPC was successfully stabilized by adding a buffer solution to the reaction solution, which established continuous analysis by HPLC using an autosampler. The DBD-TPC calibration curve was linear in the range of $0.25-250 \mathrm{nmol} / \mathrm{mL}$ (final concentration) and showed a wide dynamic range. The lower limit of detection was $0.205 \mathrm{nmol} / \mathrm{mL}$ in TPC standard. The proposed method was successfully applied to the measurement of TPC in daikon-oroshi. The results reveal the possibility of determining the variation of TPC level in processed foods containing radish. We concluded that the proposed method is useful for evaluating the quality of processed radish products.
\end{abstract}

Keywords: 2-thioxo-3-pyrrolidinecarbaldehyde; 4-(N,N-dimethylaminosulfonyl)-7-hydrazino-2,1,3benzoxadiazole; daikon-oroshi; takuan-zuke; aldehyde analysis

\section{Introduction}

Daikon (Raphanus sativus L.) belongs to the plant family Brassicaceae and is among the most common cruciferous vegetables in Japan. Cruciferous vegetables produce distinctive pungent flavored compounds called isothiocyanates (ITCs) upon the destruction of their cells. 4-Methylthio-3-butenyl isothiocyanate (MTB-ITC) is most abundant $(>90 \%)$ in white radish $[1,2]$. Studies on the physiological 
function of MTB-ITC have revealed various effects including antimutagenicity and cancer prevention [3-5]. However, MTB-ITC is unstable compared with other ITCs in the aqueous phase and is converted to 2-thioxo-3-pyrrolidinecarbaldehyde (TPC) [6]. Consequently MTB-ITC exerts complex effects, including those through degradation products, such as TPC. TPC has been reported to have antibacterial activities against bacteria causative of food poisoning and microorganisms associated with the development of caries, as well as a range of functions such as antimutagenicity [7-10]. In addition, TPC is an important intermediate in the yellowing of takuan-zuke pickle (i.e., salted radish root), which is a popular Japanese pickle [11,12], and is converted to a more stable form, 1-(2-thioxopyrrolidine-3-yl)-1,2,3,4-tetrahydro- $\beta$-carboline-3-carboxylic acid (TPCC), in the salting process by reacting with tryptophan [13].

The establishment of a method for measuring TPC is important for evaluating not only the quality of takuan-zuke but also that of other processed radish products. However, since TPC has high reactivity, it is difficult to analyze it quantitatively in a robust manner. Therefore, HPLC analysis following pre-column derivatization was carried out, focusing on the aldehyde group of TPC. In preliminary experiments, a derivatization method using 2,4-dinitrophenylhydrazine (DNPH) reagent, which is widely used for aldehyde analysis, was examined. It was difficult to analyze at low concentrations because DNPH-TPC has low stability at $4{ }^{\circ} \mathrm{C}$ and it contains many impurities derived from reagents. Therefore, a method of fluorescence derivatization analysis by 4-(N,N-dimethylaminosulfonyl)-7-hydrazino-2,1,3-benzoxadiazole (DBD-H), used for high-selectivity and -sensitivity analysis of aldehyde, was investigated [14].

In this study, we aimed to establish optimal conditions for a highly sensitive and stable analytical HPLC method for derivatizing TPC contained in takuan-zuke with DBD-H (Figure 1).
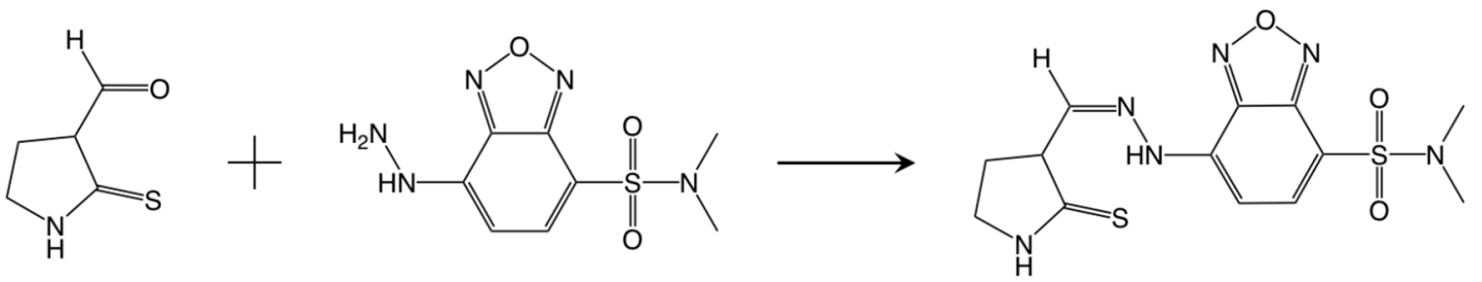

Figure 1. Fluorescence labeling reaction of 2-thioxo-3-pyrrolidinecarbaldehyde (TPC) with DBD-H.

\section{Materials and Methods}

\subsection{Materials and Reagents}

Radish roots were purchased from a market in Gunma, Japan. TPC was prepared by previously described methods [15]. 4-(N,N-dimethylaminosulfonyl)-7-hydrazino-2,1,3-benzoxadiazole (DBD-H) was purchased from Tokyo Chemical Industry (Tokyo, Japan). Trifluoroacetic acid (TFA), acetonitrile, and anisaldehyde, were purchased from Kanto Chemical (Tokyo, Japan).

\subsection{Fluorescent Labeling with $D B D-H$}

\subsubsection{TPC Standard Solution}

In brief, $250 \mu \mathrm{L}$ of $0.1 \%$ DBD in acetonitrile, $250 \mu \mathrm{L}$ of $0.2 \mathrm{mM}$ anisaldehyde in acetonitrile, and $250 \mu \mathrm{L}$ of $0.1-1.0 \%$ TFA in $20 \%(v / v)$ acetonitrile were added to $250 \mu \mathrm{L}$ of $0.1 \mathrm{mM}$ TPC in acetonitrile. The reaction mixture was shaken and incubated at $25^{\circ} \mathrm{C}$ for $0-120 \mathrm{~min}$. After ice-cooling for $5 \mathrm{~min}$, the mixture was centrifuged at $20,630 \times \mathrm{g}$ for $1 \mathrm{~min}$. To $100 \mu \mathrm{L}$ of the upper layer, $25 \mu \mathrm{L}$ of $500 \mathrm{mM}$ Mcllvaine buffer ( $\mathrm{pH} 4-6$ ) and $25 \mathrm{mg}$ of $\mathrm{NaCl}$ were added. The mixture was shaken to separate the acetonitrile phase from the aqueous phase, and $5 \mu \mathrm{L}$ of acetonitrile phase was injected into the HPLC system. 


\subsubsection{Takuan-zuke Sample}

In brief, $250 \mu \mathrm{L}$ of $0.1 \%$ DBD in acetonitrile, $250 \mu \mathrm{L}$ of $0.2 \mathrm{mM}$ anisaldehyde in acetonitrile, $250 \mu \mathrm{L}$ of acetonitrile, and $250 \mu \mathrm{L}$ of $1.0 \%$ TFA in $20 \%(v / v)$ acetonitrile were added to $10-50 \mathrm{mg}$ of takuan-zuke lyophilized powder. The reaction mixture was analyzed in accordance with the method described above.

\subsection{Analysis of TPC and MTB-ITC Contained in Daikon-oroshi (Grated Radish)}

\subsubsection{Preparation of Grated Radish Juice}

Fresh radish was grated with a radish grater and then immediately filtered with a tea strainer to obtain squeezed juice. This procedure was carried out under ice-cold conditions. The sample was prepared using a 100-mm long portion (total length $300 \mathrm{~mm}$ ) obtained from the tip of the radish root.

\subsubsection{Fluorescent Labeling of TPC from Grated Radish Juice with DBD-H}

In brief, $250 \mu \mathrm{L}$ of $0.1 \%$ DBD in acetonitrile, $250 \mu \mathrm{L}$ of $0.2 \mathrm{mM}$ anisaldehyde in acetonitrile, $250 \mu \mathrm{L}$ of $1.0 \%$ TFA in acetonitrile, $50 \mu \mathrm{L}$ of acetonitrile, and $150 \mu \mathrm{L} \mathrm{H} \mathrm{H}_{2} \mathrm{O}$ were added to $50 \mu \mathrm{L}$ of grated radish juice. The reaction mixture was analyzed in accordance with the method described above.

\subsubsection{Gas Chromatography Analysis of MTB-ITC in Grated Radish Juice}

In brief, $200 \mu \mathrm{L}$ of $0.5 \mathrm{mM}$ allyl isothiocyanate in ethyl acetate was added to $200 \mu \mathrm{L}$ of grated radish juice. The mixture was shaken vigorously to dissolve released MTB-ITC into ethyl acetate. After centrifugation $(20,630 \times g, 1 \mathrm{~min})$, the layer of ethyl acetate was recovered into a vial and then dehydrated with anhydrous sodium sulfate. The ethyl acetate solution containing isothiocyanates was analyzed by gas chromatography (GC).

\subsection{HPLC System and Conditions}

The HPLC system consisted of an Agilent 1200 pump (Agilent Technologies, Palo Alto, CA, USA), a 1200 auto sampler with a $100 \mu \mathrm{L}$ loop, 1100 column compartment, 1260 photodiode array detector, and 1100 fluorescence detector with an $8 \mu \mathrm{L}$ flow cell. Chromatographic separation was performed on a Poroshell HPH-C18 $(100 \times 3.0 \mathrm{~mm} ø, 2.7 \mu \mathrm{m}$; Agilent Technologies $)$. These analyzes can be separated by a general reversed phase column (ex. Poroshell EC-C18; Agilent Technologies). The flow rate was $0.85 \mathrm{~mL} / \mathrm{min}$. The column temperature was set to $40^{\circ} \mathrm{C}$. Elution was achieved using a gradient of two eluents: $\mathrm{H}_{2} \mathrm{O}$ as eluent $\mathrm{A}$ and acetonitrile as eluent $\mathrm{B}$ without an ion pair additive and/or buffer. The gradient program was as follows: $25 \% \mathrm{~B}$ rising to $73 \% \mathrm{~B}$ at $5.5 \mathrm{~min}$, further rising to $100 \% \mathrm{~B}$ at $5.6 \mathrm{~min}$, and remaining at $100 \% \mathrm{~B}$ to $5.99 \mathrm{~min}$. Finally, the separation column was equilibrated using $25 \%$ B from 5.99 to $8.0 \mathrm{~min}$. Fluorescence detection was performed with exciting at $450 \mathrm{~nm}$ and emitting at $565 \mathrm{~nm}$ as reported previously [14]. Anisaldehyde was used as internal standard.

\subsection{GC System and Conditions}

Gas chromatography was carried out on an Agilent Technologies 7890A GC system (Agilent Technologies). Chromatographic separation was performed on a DB-23 (30 m $\times 0.25 \mathrm{~mm} \varnothing$, film thickness $0.25 \mu \mathrm{m}$; Agilent Technologies). The carrier gas was helium with a flow rate of $1 \mathrm{~mL} / \mathrm{min}$. The column temperature was programmed as follows: $70^{\circ} \mathrm{C}$ held for $1 \mathrm{~min}$, increased to $200{ }^{\circ} \mathrm{C}$ at a rate of $15{ }^{\circ} \mathrm{C} / \mathrm{min}$, then ramped to $250^{\circ} \mathrm{C}$ at a rate of $30^{\circ} \mathrm{C} / \mathrm{min}$, and held for $1 \mathrm{~min}$, giving a total run time of $10.3 \mathrm{~min}$. Detection was carried out with a flame ionization detector. Allyl isothiocyanate was used as an internal standard. 


\section{Results and Discussion}

\subsection{Effects of Acetonitrile Concentration and Reaction Time on Extraction and Reaction Efficiency}

We investigated the optimization conditions for TPC analysis by an aldehyde derivatization method with DBD-H. Figure 2 shows a typical chromatogram obtained from the standard solution of TPC after labeling with DBD-H.

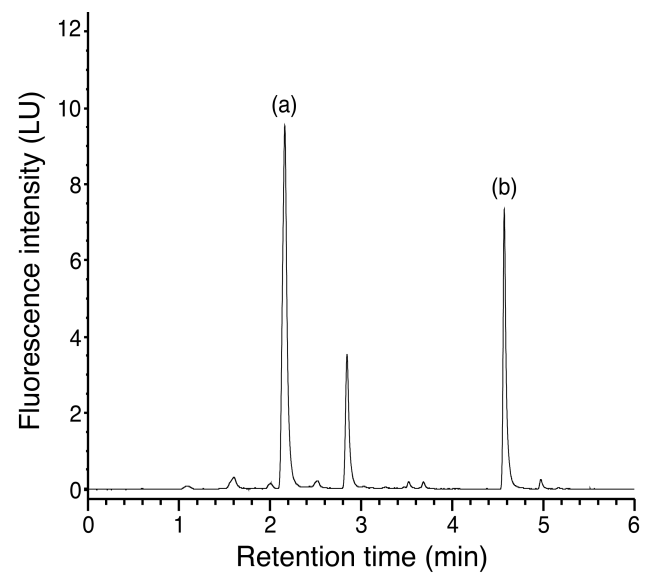

Figure 2. Chromatogram of standard solution of TPC $(0.1 \mathrm{mM})$. This result was obtained by the analysis of the standard solution of $0.1 \mathrm{mM}$ TPC (a); and $0.2 \mathrm{mM}$ anisaldehyde (b) using HPLC.

Uzu et al. reported that the reactivity of DBD-H increased in proportion to the concentration of acetonitrile [14]. The reactions of TPC and anisaldehyde (I.S.) with DBD-H in 50-100\% acetonitrile were investigated. Reaction conditions were $0.025 \%$ DBD-H and $0.025 \%$ TFA for 60 to $120 \mathrm{~min}$ at $25{ }^{\circ} \mathrm{C}$. As shown in Figure 3A, the derivative reaction lasting for $2 \mathrm{~h}$ occurred at a sufficient level for all acetonitrile concentrations, but the reaction for $1 \mathrm{~h}$ was insufficient for $50 \%$ to $60 \%$ acetonitrile.

Next, we investigated solvent conditions suitable for extraction and reaction using takuan-zuke samples, TPC, and MTB-ITC standard solution. The MTB-ITC standard was used to confirm TPC formation during the reaction. The effect of acetonitrile concentration is shown in Figure 3B. The extraction and reaction efficiency were constant in the range of 50-90\% acetonitrile for the TPC standard solution and in the range of 70-90\% for the takuan-zuke sample. Conversion of MTB-ITC to TPC was observed during a derivatization reaction with acetonitrile at a concentration of $70 \%$ or less. It was suggested that TPC was generated from residual MTB-ITC when the acetonitrile concentration was less than $80 \%$, and that extraction and reaction efficiency decreased above an acetonitrile concentration of $90 \%$; thus, $80 \%$ acetonitrile was selected as the optimal condition for subsequent analyses.

\subsection{Effects of Trifluoroacetic Acid Concentration on Reaction Efficiency of DBD-H and Stability of DBD-TPC}

Uzu et al. reported that DBD-H becomes more reactive with increasing TFA concentration and reacts not only with aldehyde groups but also with ketones. Figure 4 shows the DBD-TPC reaction efficiency in the presence of $0.025-0.25 \%$ TFA. Derivatized efficiency increased in proportion to the TFA concentration; the reaction with $0.25 \%$ TFA was completed by $60 \mathrm{~min}$.

The area ratio was calculated from the $0.05 \mathrm{mM}$ internal standard and the TPC area value. 

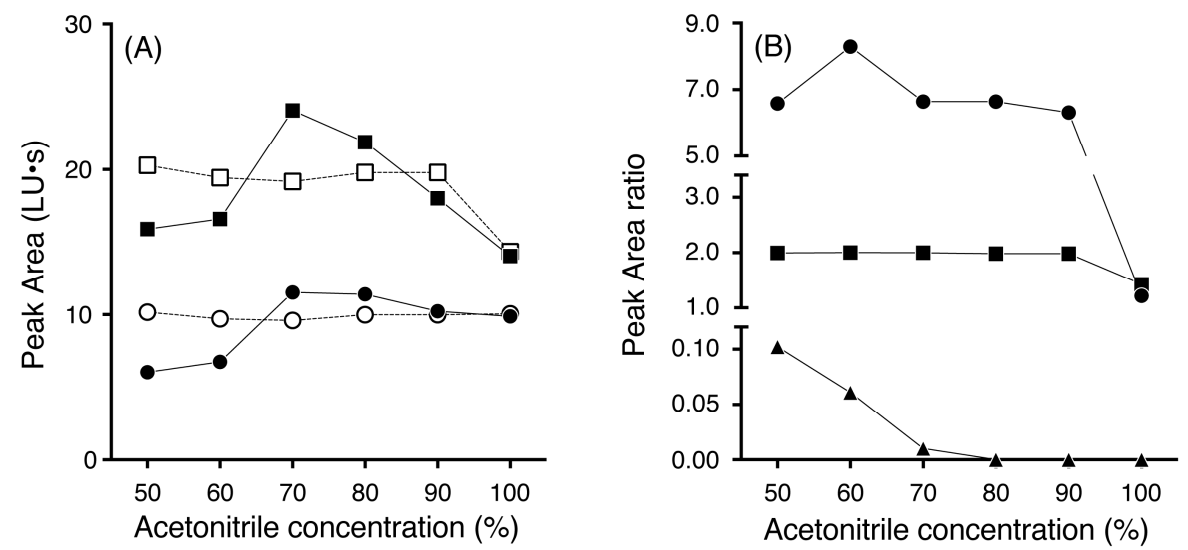

Figure 3. Effects of acetonitrile concentrations on the reaction efficiency of DBD-H. The effect of acetonitrile concentration and reaction time on the DBD-H derivatization reaction (A). Symbols: $\bigcirc 0.05 \mathrm{mM}$ anisaldehyde and $\square 0.025 \mathrm{mM}$ TPC reacted for $60 \mathrm{~min} ; \bullet 0.05 \mathrm{mM}$ anisaldehyde and

$0.025 \mathrm{mM}$ TPC reacted for $120 \mathrm{~min}$. The effect of acetonitrile concentration on extraction and DBD-H derivatization reaction (B). Symbols: $\bullet 10 \mathrm{mg}$ of takuan-zuke, $0.025 \mathrm{mM}$ TPC, $\boldsymbol{\Delta} 0.025 \mathrm{mM}$ MTB-ITC.
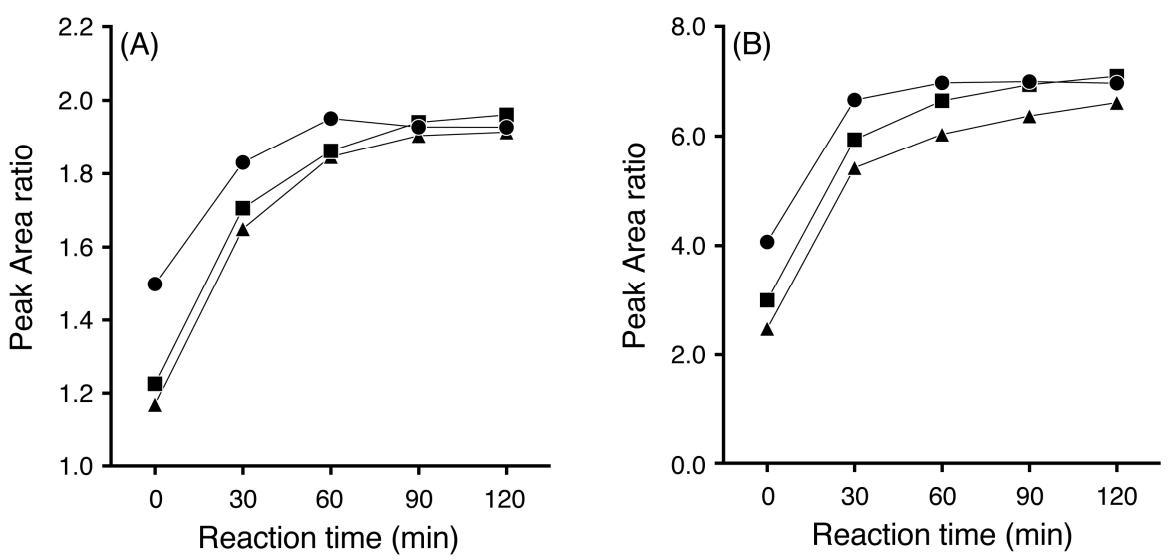

Figure 4. Effects of trifluoroacetic acid (TFA) concentrations on the area ratio of TPC. $0.1 \mathrm{mM} \mathrm{TPC}$ standard solution (A); and $10 \mathrm{mg}$ of takuan-zuke sample (B). Symbols: $\bullet 0.25 \%$ TFA, $0.05 \%$ TFA, $\Delta 0.025 \%$ TFA.

The level of DBD-TPC decreased over time during storage at $4{ }^{\circ} \mathrm{C}$ for $16 \mathrm{~h}$, while that of DBD-anisaldehyde was stable. Matsuoka et al. reported that TPC is stable around $\mathrm{pH} 5$ [9]. It was assumed that the stability of DBD-TPC was related to the structure of TPC under low $\mathrm{pH}$. It was suggested that the $\mathrm{pH}$ buffering ability of takuan-zuke sample influences the stability of DBD-TPC.

The area ratio was calculated from the $0.05 \mathrm{mM}$ internal standard and the TPC area value.

\subsection{Effect of $p H$ on Stability of DBD-TPC}

The effect of $\mathrm{pH}$ on the stability of DBD-TPC at $4{ }^{\circ} \mathrm{C}$ was confirmed by adding a Mcllvaine buffer of $\mathrm{pH} 4,5$, and 6 to the derivatized sample immediately after the reaction. As shown in Figure 5, the DBD-TPC residual proportion in the TPC standard solution was $86 \%$ when no buffer was added. In contrast, DBD-TPC with buffer was stable. Similar results were obtained with the takuan-zuke sample. From these results, DBD-TPC was stabilized by adding $\mathrm{pH} 5-6$ buffer.

The optimal reaction conditions for TPC derivatization were set as $80 \%$ acetonitrile solvent containing $0.25 \%$ TFA for $60 \mathrm{~min}$ at $25^{\circ} \mathrm{C}$. Thereafter, Mcllvaine buffer solution (pH 5) was added to the reaction mixture and analysis was performed with an HPLC system equipped with a thermostated autosampler and a fluorescence detector. 

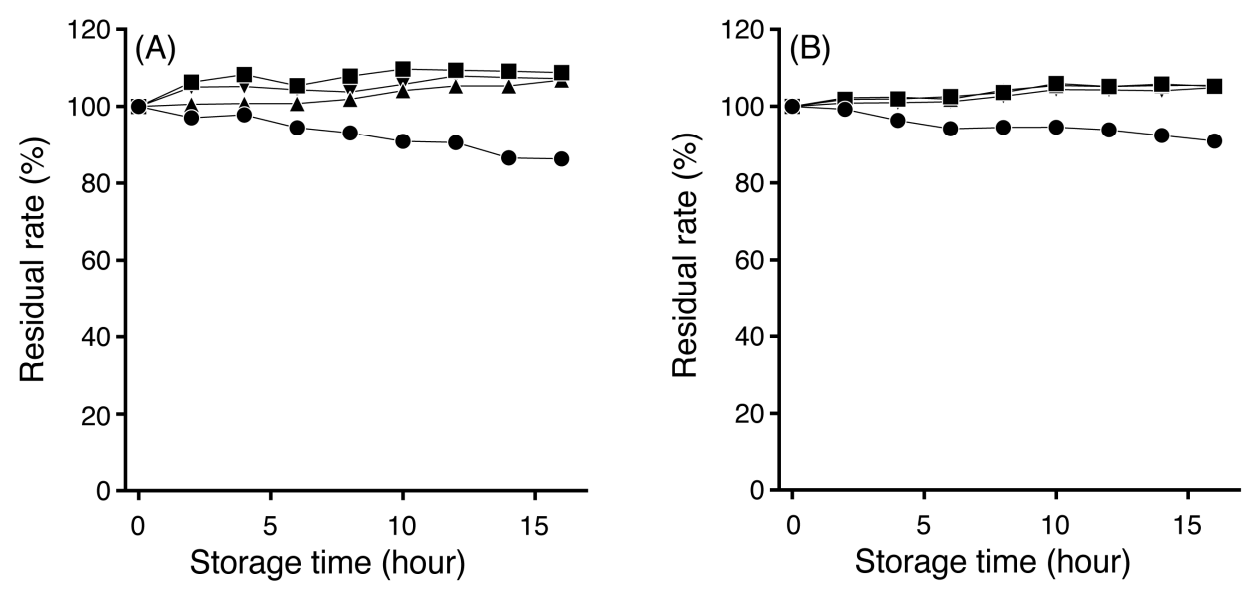

Figure 5. Effects of buffer $\mathrm{pH}$ on the stability of TPC. (A) $0.1 \mathrm{mM}$ TPC standard solution; and (B) $10 \mathrm{mg}$ of takuan-zuke sample. Symbols: $\bullet$ No addition of buffer, $\boldsymbol{\square}$ p4 McIlvaine buffer, $\boldsymbol{\Delta}$ pH5 McIlvaine buffer, $\mathbf{\nabla}$ pH6 Mcllvaine buffer. The residual ratio was represented by the peak area ratio when the peak area value immediately after the reaction was taken as $100 \%$.

\subsection{Verification of Sensitivity}

Figure 6 shows the linearity of DBD-TPC under optimized reaction conditions. The calibration curve of TPC was linear $\left(y=1.34 x-0.06, r^{2}=1.000\right)$ in the range of 0.25 to $250 \mathrm{nmol} / \mathrm{mL}$ (final concentration), with a wide dynamic range. The detection limit (signal-to-noise ratio $=3$ ) of the proposed method was $0.205 \mathrm{nmol} / \mathrm{mL}$.

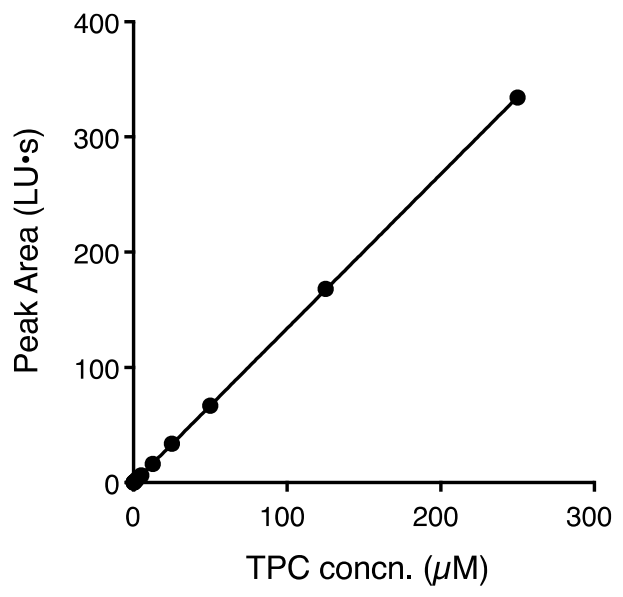

Figure 6. Linearity of TPC concentration in DBD-H fluorescent labeling.

\subsection{Quantification of TPC and MTB-ITC in Grated Radish Juice}

Time-dependent changes in TPC and MTB-ITC in grated radish juice were analyzed (Figure 7). MTB-ITC was rapidly formed during radish grinding and decreased in a time-dependent manner from the maximum concentration of $213.0 \mathrm{nmol} / 50 \mu \mathrm{L}$ of grated juice. The TPC in grated radish juice increased with time; the concentration was $0.5-160.0 \mathrm{nmol} / 50 \mu \mathrm{L}$. The results showed that about $75 \%$ of MTB-ITC was converted to TPC in only $120 \mathrm{~min}$. In addition, the cumulative value of MTB-ITC and TPC after $120 \mathrm{~min}$ was $182.4 \mathrm{nmol} / 50 \mu \mathrm{L}$, which was $85 \%$ of the initial value of MTB-ITC. A strong negative correlation between TPC and MTB-ITC was revealed $\left(y=-1.20 x+211, r^{2}=0.997\right)$ in linear regression analysis. These results revealed that TPC is the major degradation product of MTB-ITC. The findings of this study show that this analytical method can be applied not only to takuan-zuke, but also to processed radish products, with potential utility for evaluating the quality of such items. 


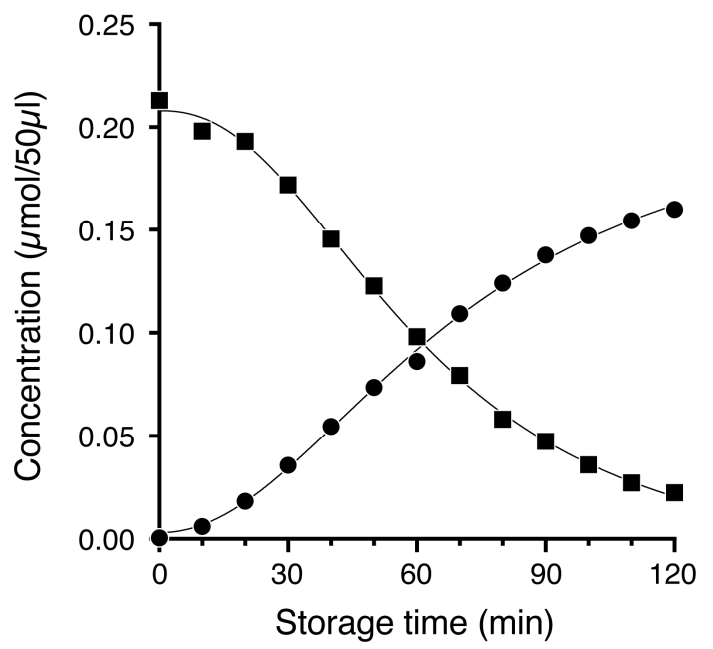

Figure 7. Changes of TPC and MTB-ITC in grated radish juice. Symbols: • TPC (HPLC method), MTB-ITC (GC method).

\section{Conclusions}

In this study, we developed a simple method to quantify TPC in takuan-zuke using HPLC by pre-column derivatization with DBD-H. In this method, extraction and fluorescence derivatization were performed in parallel to reduce the loss of TPC and enable stable analysis. This approach enabled the determination of the variation of TPC amounts in processed foods using radish. We considered this method to be useful for evaluating the quality of processed radish products.

Acknowledgments: The authors would like to thank the language editing service Enago for the English language review.

Author Contributions: The experimental design was constructed and supervised by T.K., K.K., W.K., A.T., and H.M. The production of samples was performed by T.K., K.K., W.K., A.T., and H.T. Instrumental analysis were performed by T.K., K.K., and W.K. The manuscript was drafted and written by T.K., and H.M.

Conflicts of Interest: The authors declare no conflict of interest.

\section{References}

1. Friis, P.A.; Kjaer, A. 4-methylthio-3-butenyl isothiocyanate, the pungent principle of radish root. Acta Chem. Scand. 1966, 20, 698-705. [CrossRef]

2. Kjr, A.; Madsen, J.O.; Maeda, Y.; Ozawa, Y.; Uda, Y. Volatiles in distillates of fresh radish of Japanese and Kenyan origin. Agric. Biol. Chem. 1978, 42, 1715-1721.

3. Nakamura, Y.; Iwahashi, T.; Tanaka, A.; Koutani, J.; Matsuo, T.; Okamoto, S.; Sato, K.; Ohtsuki, K. 4-(methylthio)-3-butenyl isothiocyanate, a principal antimutagen in daikon (Raphanus sativus; Japanese white radish). J. Agric. Food Chem. 2001, 49, 5755-5760. [CrossRef] [PubMed]

4. Okamura, T.; Umemura, T.; Inoue, T.; Tasaki, M.; Ishii, Y.; Nakamura, Y.; Park, E.Y.; Sato, K.; Matsuo, T.; Okamoto, S.; et al. Chemopreventive effects of 4-methylthio-3-butenyl isothiocyanate (Raphasatin) but not curcumin against pancreatic carcinogenesis in hamsters. J. Agric. Food Chem. 2013, 61, 2103-2108. [CrossRef] [PubMed]

5. Yamasaki, M.; Omi, Y.; Fujii, N.; Ozaki, A.; Nakama, A.; Sakakibara, Y.; Suiko, M.; Nishiyama, K. Mustard oil in "Shibori Daikon" a variety of Japanese radish, selectively inhibits the proliferation of H-ras-transformed 3Y1 cells. Biosci. Biotechnol. Biochem. 2009, 73, 2217-2221. [CrossRef] [PubMed]

6. Uda, Y.; Ozawa, Y.; Ohshima, T.; Kawakishi, S. Identification of enolated 2-thioxo-3-pyrrolidinecarbaldehyde, a new degradation product of 4-methylthio-3-butenyl isothiocyanate. Agric. Biol. Chem. 1990, 54, 613-617.

7. Hashimoto, K.; Yanagi, K.; Fukushima, K.; Uda, Y. Effect of 3-hydroxymethylene-2-thioxopyrrolidine on growth of two species of mutans streptococci and their in vitro plaque formation. Int. J. Antimicrob. Agents 2001, 17, 97-102. [CrossRef] 
8. Uda, Y.; Matsuoka, H.; Shima, H.; Kumagami, H.; Maeda, Y. Antimicrobial activity of water-soluble products derived from radish mustard oil and identification of an active component therein. Nippon Shokuhin Kougyo Gakkaishi 1993, 40, 801-806. [CrossRef]

9. Matsuoka, H.; Takahashi, A.; Yanagi, K.; Uda, Y. Antimicrobial action of 2-thioxo-3-pyrrolidinecarbardehyde, a major thiolactam compound generated from the pungent principle of radish in an aqueous medium. Food Sci. Technol. Int. Tokyo 1997, 3, 353-356. [CrossRef]

10. Uda, Y.; Hayashi, H.; Takahashi, A.; Shimizu, A. Mutagenic and antimutagenic property of 3-hydroxymethylene2-thioxopyrrolidine, a major product generating from pungent principle of radish. LWT-Food Sci. Technol. 2000, 33, 37-43. [CrossRef]

11. Ozawa, Y.; Uda, Y.; Kawakishi, S. Generation of $\beta$-carboline derivative, the yellowish precursor of processed radish roots, from 4-methylthio-3-butenyl isothiocyanate and L-tryptophan. Agric. Biol. Chem. 1990, 54, 1849-1851.

12. Matsuoka, H.; Takahashi, A.; Ozawa, Y.; Yamada, Y.; Uda, Y.; Kawakishi, S. 2-[3-(2-thioxopyrrolidin-3ylidene)methyl]-tryptophan, a novel yellow pigment in salted radish roots. Biosci. Biotechnol. Biochem. 2002, 66, 1450-1454. [CrossRef] [PubMed]

13. Takahashi, A.; Yamada, T.; Uchiyama, Y.; Hayashi, S.; Kumakura, K.; Takahashi, H.; Kimura, N.; Matsuoka, H. Generation of the antioxidant yellow pigment derived from 4-methylthio-3-butenyl isothiocyanate in salted radish roots (takuan-zuke). Biosci. Biotechnol. Biochem. 2015, 79, 1512-1517. [CrossRef] [PubMed]

14. Uzu, S.; Kanda, S.; Imai, K.; Nakashima, K.; Akiyama, S. Fluorogenic reagents: 4-aminosulphonyl-7hydrazino-2,1,3-benzoxadiazole, 4-(N,N-dimethylaminosulphonyl)-7-hydrazino-2,1,3-benzoxadiazole and 4-hydrazino-7-nitro-2,1,3-benzoxadiazole hydrazine for aldehydes and ketones. Analyst 1990, 115, 1477-1482. [CrossRef]

15. Matsuoka, H.; Uda, Y.; Mitani, K.; Yoneyama, K. Easy preparation method for 2-thioxopyrrolidine derivatives including 3-hydroxymethylene-2-thioxopyrrolidine, an antimicrobial degradation product of radish pungent principle, via (E,Z)-4-methoxy-3-butenyl isothiocyanate. Biosci. Biotechnol. Biochem. 1996, 60, 914-915. [CrossRef] [PubMed]

(C) 2017 by the authors. Licensee MDPI, Basel, Switzerland. This article is an open access article distributed under the terms and conditions of the Creative Commons Attribution (CC BY) license (http:/ / creativecommons.org/licenses/by/4.0/). 